\title{
FREE BURNSIDE SEMIGROUPS *
}

\author{
Alair Pereira do Lago $^{1}$ And Imre Simon ${ }^{1}$
}

\begin{abstract}
This paper surveys the area of Free Burnside Semigroups. The theory of these semigroups, as is the case for groups, is far from being completely known. For semigroups, the most impressive results were obtained in the last 10 years. In this paper we give priority to the mathematical treatment of the problem and do not stress too much neither motivation nor the historical aspects. No proofs are presented in this paper, but we tried to give as many examples as was possible.
\end{abstract}

Mathematics Subject Classification. 20M05, 20F50.

\section{INTRODUCTION}

This paper surveys the area of relatively Free Burnside Semigroups which satisfy the equation $x^{n}=x^{n+m}$. This is a classical problem area in Algebra and the theory for semigroups was much slower to develop than the corresponding theory for groups, where several Russian schools of thought contributed an immense amount of knowledge. The theory of these semigroups, as is the case for groups, is far from being completely known. However, for semigroups tremendous progress was achieved in the last 10 years.

The theory of Burnside semigroups progressed in bursts of discoveries. Work of Thue in the beginning of the century implies that these semigroups are infinite for $n \geq 2$ and $m \geq 1$ if they have at least three generators or for $n \geq 3$ and $m \geq 1$ if they have two generators. In the 50's Green, Rees and McLean advanced our knowledge for the case $n=1$. In the early 70's some advances were made in the school of Brzozowski and then the problem became dormant for almost 20 years. The most impressive results were obtained in the last 10 years by

\footnotetext{
* Partially supported by the following institutions/grants: University of São Paulo - DIMACS - FAPESP process 2000/08039-3 - Alfred P. Sloan Foundation Grant number 99-10-8 - National Science Foundation NSF DBI 99-82983 - CNPq processes 523557/96$5(N V)$ and 465901/00-0 $(N V)$.

1 Instituto de Matemática e Estatística, Universidade de São Paulo, Rua do Matão 1010, 05508-090 São Paulo, SP, Brazil; e-mail: alair@ime.usp.br \& is@ime.usp.br
} 
de Luca, Varricchio, McCammond, Guba, Kaďourek, Polák and Pereira do Lago in the references cited at the end of this paper.

In this paper we give priority to the mathematical treatment of the problem and do not stress much neither motivation nor the historical aspects. These can be found in [14]. No proofs are presented in this paper, those can be found in the references. We tried to give as many examples as was possible.

The ultimate unifying theme of this subject are the discoveries of finitary properties of the structures we investigate. We are also interested in algorithmic aspects and the effectivity of our arguments and procedures. Loosely speaking, there seems to arise a hierarchy in this direction: the finiteness of the whole, the finiteness of certain parts, including the rationality of the congruence classes (Brzozowski's conjecture). We are also interested in the effective solvability of the word problem for these semigroups. We will try to keep focused on these objectives throughout the paper.

\section{The Free Burnside SEMIGRouP}

Let $A$ be any alphabet and suppose that $n$ and $m$ are integers such that $n \geq 1$ and $m \geq 1$. Let $\pi=\left\{\left(x^{n+m}, x^{n}\right) \mid x \in A^{+}\right\}$and let $\sim$ be the smallest congruence on $A^{*}$ that contains $\pi$. We define the free Burnside monoid satisfying $x^{n}=x^{n+m}$ generated by $A$ to be the quotient monoid $\mathcal{M} \stackrel{\text { def }}{=} A^{*} / \sim$. We define the canonical morphism ${ }^{\sim}$

$$
\sim: A^{*} \longrightarrow \mathcal{M}
$$

that maps a word $w \in A^{*}$ on its congruence class $\widetilde{w} \in \mathcal{M}$. Note that $w \sim w^{\prime} \Longleftrightarrow$ $\widetilde{w}=\widetilde{w^{\prime}}$. We define the free Burnside semigroup satisfying $x^{n}=x^{n+m}$ generated by $A$ to be $\mathcal{M} \backslash\{\widetilde{1}\}$.

Consider the Green relations $\mathcal{J}, \mathcal{D}, \mathcal{R}, \mathcal{L}$ and $\mathcal{H}$ as defined in [24] and recall that an element $x$ of a monoid is called idempotent if $x=x x$, it is called regular if there exists an element $y$ such that $x=x y x$ and it is called irregular otherwise. For every element $x \in \mathcal{M}$ we have that $x^{n}=x^{n+m}$. This in particular implies that $\mathcal{M}$ is a torsion monoid and, consequently, $\mathcal{J}=\mathcal{D}$. If $n=m=1$, the free Burnside monoid satisfying $x^{n}=x^{n+m}$ generated by $A$ is also called the free band generated by $A$ or the free idempotent monoid generated by $A$.

In the next four sections we will see definitions and results that are valid for any values $n \geq 1$ and $m \geq 1$. In Section 2 we will see the description of an important property of the structure of the $\mathcal{R}$-classes of $\mathcal{M}$; namely, that the frame of the $\mathcal{R}$-classes is a tree. In Section 5 we will see two theorems that give a precise description of the internal structure of the $\mathcal{H}$-classes: the maximal groups of $\mathcal{M}$ are free Burnside groups. To reach these results we shall introduce new techniques for the study of $\mathcal{M}$. The basis of these techniques is a concept to be defined in Section 3: the fundamental graph of a $\mathcal{D}$-class. We will also need some properties of certain free categories defined on the fundamental graph, these topics will be dealt with in Section 4. 
In Section 6 we will define a new relation $\Sigma$ which generates the same congruence as $\pi$, and which leads to an important technique to be used in the study of $\mathcal{M}$, whenever $\Sigma$ is stable (to be defined there). In Section 8, besides other properties valid for the case where $\Sigma$ is stable, we will present an effective characterization of the fundamental graph and also a solution of the word problem.

Our study of the free Burnside monoids is subdivided in three cases. Each case uses different techniques and different sets of properties are achieved. In Section 7 we describe the properties known for the cases in which $n=1$. In Section 8 we present the case where $\Sigma$ is stable. This is the case where we have the more complete knowledge of the free Burnside monoid and it occurs whenever $n \geq 3$. Stability might possibly occur also for $n=2$ and $m=1$. In Section 9 we present what is known for $n=2$. We remark that the least understood case is $n=2$ and $m=1$.

\section{THE THEOREM ON MARKERS}

The morphism $\sim: A^{*} \longrightarrow \mathcal{M}$ and the Green relations in $\mathcal{M}$ induce other relations $\mathcal{J}^{\prime}, \mathcal{R}^{\prime}, \mathcal{L}^{\prime}, \mathcal{H}^{\prime}$ and $\mathcal{D}^{\prime}$ in $A^{*}$ in the following way:

$$
x \rho^{\prime} y \Longleftrightarrow \widetilde{x} \rho \widetilde{y}, \text { for } \rho \in\{\mathcal{J}, \mathcal{R}, \mathcal{L}, \mathcal{H}, \mathcal{D}\}
$$

These relations will be called the Green-induced relations by the morphism ${ }^{\text {or }}$ simply Green-induced relations. These relations are equivalences, because so are the Green relations defined on $\mathcal{M}$.

Let $w \in A^{+}$. We define $\epsilon_{R}(w)$ the $\mathcal{R}$-entrance of $w$ to be the shortest prefix of $w$ that belongs to the $\mathcal{R}^{\prime}$-class of $w$ and we say that $w$ is an $\mathcal{R}$-entrance if $\epsilon_{R}(w)=w$. Note that $y$ is the $\mathcal{R}$-entrance of $w$ if and only if $y$ is an $\mathcal{R}$-entrance and $w \mathcal{R}^{\prime} y \in \operatorname{Pref}(w)$, where $\operatorname{Pref}(w)$ denotes the set of prefixes of $w$. Furthermore, any prefix $u$ of $w$ not shorter than $\epsilon_{R}(w)$ is in the $\mathcal{R}^{\prime}$-class of $w$. In a dual way, we define $\epsilon_{L}(w)$ the $\mathcal{L}$-entrance of $w$ to be the shortest suffix of $w$ that belongs to the $\mathcal{L}^{\prime}$-class of $w$ and we say that $w$ is an $\mathcal{L}$-entrance if $\epsilon_{L}(w)=w$.

In 1970 I. Simon [34] studied the free Burnside monoid satisfying $x^{2}=x^{3}$ generated by a set of two generators and tried to get some finiteness properties for the monoid. In fact, it had just been discovered that this monoid was infinite, by work of Brzozowski et al. [4]. However, he was able to show some properties already known to hold in the free band and the most important among them is Theorem $2.1^{2}$ :

Theorem 2.1 (theorem on Markers). Take integers $n \geq 1$ and $m \geq 1$, let $\mathcal{M}$ be the free Burnside monoid satisfying $x^{n}=x^{n+m}$ generated by an alphabet $A$ and consider the Green-induced relations by the canonical morphism ${ }^{\sim}: A^{*} \longrightarrow \mathcal{M}$.

\footnotetext{
${ }^{2}$ For $x, y \in A^{*}, x y^{-1}$ is $z$ if $x=z y$ for some $z \in A^{*}$ and it is undefined otherwise. $x^{-1} y$ is defined dually.
} 
Let $w, w^{\prime} \in A^{+}$be two words such that $w \mathcal{R}^{\prime} w^{\prime}$ and let $u$ and $u^{\prime}$ be their respective $\mathcal{R}$-entrances. Then:

- $u \sim u^{\prime}$

- the last letter of $u$ is the same as the last letter of $u^{\prime}$;

- $u a^{-1} \sim u^{\prime} a^{-1}$, where $a$ is the last letter of $u$ and of $u^{\prime}$.

We recall that the frame of the $\mathcal{R}$-classes is a directed graph where the vertices are the $\mathcal{R}$-classes and there exists an edge with label $g$ from an $\mathcal{R}$-class $R$ to a different $\mathcal{R}$-class $R^{\prime}$ if and only if there exist $x \in R$ and a generator $g$ such that $x g \in R^{\prime}$. If the $\mathcal{R}$-entrance of a word $w \in A^{+}$is $u a$, where $a \in A$, then Theorem 2.1 implies that the $\mathcal{R}$-class of $\widetilde{w}$ receives a unique edge in the frame and this edge comes from the $\mathcal{R}$-class of $\widetilde{u}$ and has label $\widetilde{a}$. Corollary 2.2 follows easily.

Corollary 2.2. The frame of the $\mathcal{R}$-classes of $\mathcal{M}$ is a tree.

\section{THE FUNDAMENTAL GRAPH}

Given words $x, y, z$ and $w$, we say that $(x, y, z)$ is a factorization of $w$ if $w=x y z$. In the set of factorizations of $w$, we define the order of the factorizations $\leq$ as follows: $(x, y, z) \leq\left(x^{\prime}, y^{\prime}, z^{\prime}\right)$ if and only if $|x| \geq\left|x^{\prime}\right|$ and $|z| \geq\left|z^{\prime}\right|$. Given a set of factorizations of $w$ incomparable with respect to the order of the factorizations, we define the order of the occurrences $\preccurlyeq:(x, y, z) \preccurlyeq\left(x^{\prime}, y^{\prime}, z^{\prime}\right)$ if and only if $|x| \leq\left|x^{\prime}\right|$.

We say that $y$ is a $\mathcal{D}$-entrance of $w$ if $y$ is a minimal factor of $w$ in the same $\mathcal{D}^{\prime}$ class of $w$; that is to say, $y$ itself is the unique factor of $y$ in the $\mathcal{D}^{\prime}$-class of $w$. We say that $w$ is a $\mathcal{D}$-entrance if $w$ is a $\mathcal{D}$-entrance of $w$. Note that $y$ is a $\mathcal{D}$-entrance if and only $y$ is a $\mathcal{D}$-entrance of some $w$. An occurrence of $\mathcal{D}$-entrance in $w$ is a factorization $(x, y, z)$ of $w$ such that $y$ is a $\mathcal{D}$-entrance of $w$. Note that a word $w$ might have more than one occurrence of $\mathcal{D}$-entrance and that the occurrences of $\mathcal{D}$-entrances of $w$ are incomparable with respect to the order of the factorizations.

We say that $y$ is a transition of $w$ if $y$ is a minimal factor of $w$ that contains exactly two $\mathcal{D}$-entrances of $w$; that is to say, if there exist exactly two different occurrences of $\mathcal{D}$-entrances of $y$ : $\left(1, \epsilon_{R}(y), \epsilon_{R}(y)^{-1} y\right)$ and $\left(y \epsilon_{L}(y)^{-1}, \epsilon_{L}(y), 1\right)$. We say that $w$ is a transition if $w$ is a transition of $w$. Again, $y$ is a transition if and only if $y$ is a transition of some $w$. A factorization $(x, y, z)$ of $w$ in which $y$ is a transition of $w$ is called an occurrence of transition of $w$ and two such occurrences are incomparable with respect to the order of the factorizations. If $\left(x_{1}, y_{1}, z_{1}\right),\left(x_{2}, y_{2}, z_{2}\right), \ldots,\left(x_{k}, y_{k}, z_{k}\right)$, for $k \geq 0$, is the sequence of occurrences of transitions of $w$ ordered by the order of the occurrences, we define the sequence of transitions of $w$ to be the sequence $y_{1}, y_{2}, \ldots, y_{k}$.

Let $D$ be any $\mathcal{D}$-class of the free Burnside semigroup $\mathcal{M} \backslash\{\widetilde{1}\}$. Let

$$
V=\{\widetilde{d} \in D \mid d \text { is a } \mathcal{D} \text {-entrance }\}
$$

and let

$$
E=\{\tilde{t} \in D \mid t \text { is a transition }\}
$$


Let $t$ be a transition such that $\widetilde{t} \in E$. Recall that the prefix $\epsilon_{R}(t)$ and the suffix $\epsilon_{L}(t)$ are the two $\mathcal{D}$-entrances of $t$ and that both $\widetilde{\epsilon_{R}(t)}$ and $\widetilde{\epsilon_{L}(t)}$ are in $V$. Thus, we define the function $\alpha: E \longrightarrow V$ by

$$
\alpha(\widetilde{t})=\widetilde{\epsilon_{R}(t)} .
$$

This definition does not depend on the particular choice of $t$ since if $t^{\prime}$ is such that $\widetilde{t^{\prime}}=\widetilde{t}$ then Theorem 2.1 implies that $\widetilde{\epsilon_{R}\left(t^{\prime}\right)}=\widetilde{\epsilon_{R}(t)}$. In a dual way, we define the function $\omega: E \longrightarrow V$ by

$$
\omega(\widetilde{t})=\widetilde{\epsilon_{L}(t)} .
$$

Again, using a dual argument, the definition does not depend on the particular choice of $t$. Now, we define $\mathbf{G}=(V, E, \alpha, \omega)$ to be the directed graph whose set of vertices is $V$, set of edges is $E$ and whose incidence functions are $\alpha$ and $\omega$. This graph $\mathbf{G}$ is called the fundamental graph of $D$ (or fundamental graph of any element of $D$ ). This definition first appeared in [13] and $\mathbf{G}$ was proved to be strongly connected. Therefore, the cyclomatic number of $\mathbf{G}$ is the cardinal $|E|-|V|+1$.

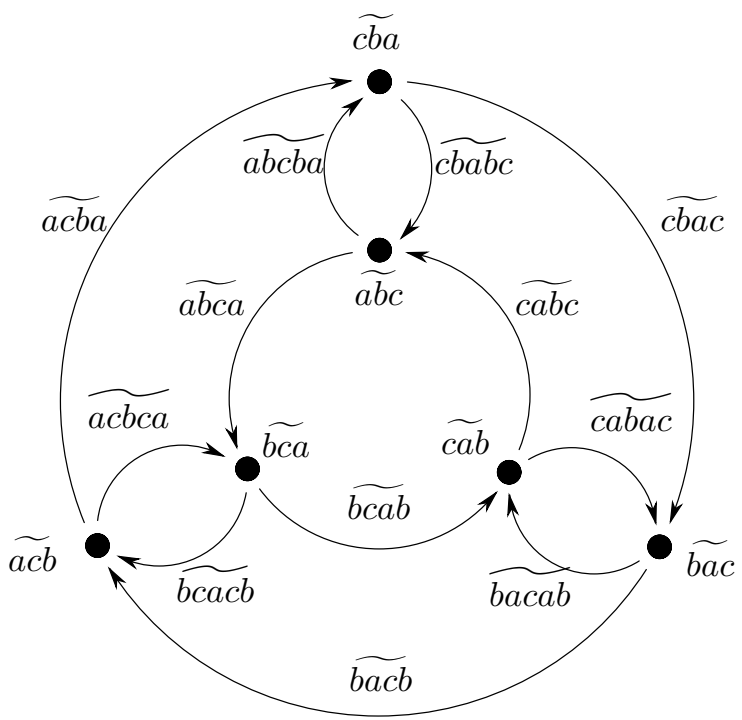

Figure 1. Fundamental graph of the $\mathcal{D}$-class of $\widetilde{a b c}$. in the free band.

In Proposition 3.1, we characterize whether or not two $\mathcal{D}$-entrances (or two transitions) are congruent.

Proposition 3.1. Two $\mathcal{D}$-entrances (or two transitions) aub and $a^{\prime} u^{\prime} b^{\prime}$, with $a, a^{\prime}, b, b^{\prime} \in A$ and $u, u^{\prime} \in A^{*}$, are congruent if and only if $a=a^{\prime}$ and $b=b^{\prime}$ and $u \sim u^{\prime}$. 
Let $w \in A^{+}$be a word, let $\mathbf{G}_{w}$ be the fundamental graph of the $\mathcal{D}$-class of $\tilde{w}$ and let $\mathbf{G}_{w}^{*}$ be the free category generated by the graph $\mathbf{G}_{w}$ (see the definition in the next section). Let $w_{1}, \ldots, w_{k}$ be the sequence of transitions of $w$ and let $y_{0}=\epsilon_{L}\left(\epsilon_{R}(w)\right)$. Note that $y_{0}=\epsilon_{L}\left(\epsilon_{R}(w)\right) \mathcal{D}^{\prime} w$ is a $\mathcal{D}$-entrance. The function $\sigma: A^{+} \longrightarrow \cup_{w \in A^{+}} \mathbf{G}_{w}^{*}$ defines the fingerprint of $w$ to be the path in $\mathbf{G}_{w}$ :

$$
\sigma(w)= \begin{cases}\left(\widetilde{w_{1}}, \widetilde{w_{2}}, \ldots, \widetilde{w_{k}}\right), & \text { if } k>0 \\ 1_{\widetilde{y_{0}}}, & \text { if } k=0\end{cases}
$$

This is a path from $\left.\epsilon_{L} \widetilde{\left(\epsilon_{R}(w)\right.}\right)$ to $\left.\epsilon_{R} \widetilde{\left(\epsilon_{L}(w)\right.}\right)$.

\section{Free Burnside Groupoid}

In this section we will use the terminology of category theory [27]. Important results connecting category theory with monoid theory can be found in Tilson's work $[37]^{3}$. It is interesting to note that the concept of category generalizes the concept of monoid while that of groupoid generalizes the concept of group.

Let $\mathbf{G}$ be a strongly connected graph whose set of vertices is $V$. Let $\mathcal{K}=\mathbf{G}^{*}$ be the free category generated by the graph $\mathbf{G}$. Loosely speaking, $\mathcal{K}$ is the set of the nonempty paths of $\mathbf{G}$ together with the empty paths $1_{v}$, one for each vertex $v$. The multiplication in $\mathcal{K}$ is a partial multiplication defined only on consecutive paths. Let $\mathcal{K}_{v}$ be the local monoid of $v$, formed by all closed paths from $v$ to $v$. Let $X$ be a set of unknowns and let $\mathcal{T} \subseteq X^{*} \times X^{*}$ be a set of monoid identities on $X$. We define the set $\mathcal{T}(\mathbf{G})=\left\{(\varphi(u), \varphi(v)) \mid(u, v) \in \mathcal{T}, z \in V, \varphi: X \longrightarrow \mathcal{K}_{z}\right\}$ and the congruence $\cong$ on $\mathcal{K}$ to be the smallest congruence that contains $\mathcal{T}(\mathbf{G})$. Notice that the above definition implies that each congruence class consists of sets of pairwise coterminal paths. We define the category $\mathcal{B}$ to be $\mathcal{B} \stackrel{\text { def }}{=} \mathcal{K} / \cong$ and for each path $p \in \mathcal{K}$ we associate its canonical projection $\widehat{p} \in \mathcal{B}$ that is the congruence class of $p$, relative to $\cong$. The objects of the category $\mathcal{B}$ are the same as those of the category $\mathcal{K}$ which are in turn the vertices of $\mathbf{G}$. Furthermore, the canonical projection from $\mathcal{K}$ onto $\mathcal{B}$ preserves the beginning and the end of every path. Since $\mathbf{G}$ is a strongly connected graph it immediately follows that $\mathcal{K}$ and $\mathcal{B}$ are strongly connected categories.

Let us suppose that $\mathcal{T}$ is such that any monoid satisfying $\mathcal{T}$ is a group. In this case, one can prove [9] that $\mathcal{B}$ is a groupoid (a category where every element is invertible), each local monoid $\mathcal{B}_{v}$ is isomorphic to the same group, which in turn satisfies the identity $x^{t}=1$ for some smallest $t \geq 1$. One example is $\mathcal{T}=\left\{\left(x^{t}, 1\right)\right\}$, for some $t \geq 1$. Notice that groups are trivial iff $t$ is 1 . The groupoid $\mathcal{B}$ thus defined is the free groupoid on $\mathbf{G}$ satisfying $\mathcal{T}$. If $\mathcal{T}$ has only the identity $x^{t}=1$ it is also called free Burnside groupoid on $\mathbf{G}$ satisfying $x^{t}=1$.

\footnotetext{
${ }^{3}$ As far as we know the earliest results in this area appeared in [5,34]. Those results were rewritten by Eilenberg [15] and in that form they probably served as an inspiration for Tilson's work. See also [35] for further historical notes.
} 
The main result [13] relative to the free Burnside groupoid is a characterization of the local groups as being free Burnside groups. This result was generalized [9] for any free groupoid on $\mathbf{G}$ satisfying $\mathcal{T}$.

Theorem 4.1. Let $\mathbf{G}$ be a strongly connected graph and let $\mathcal{T}$ be a set of monoid identities such that any monoid satisfying $\mathcal{T}$ is also a group. Let $\mathcal{B}$ be the free groupoid on $\mathbf{G}$ satisfying $\mathcal{T}$. Then, the local groups $\mathcal{B}_{v}$, for $v \in V(\mathbf{G})$, are all isomorphic to a free group satisfying $\mathcal{T}$. Furthermore, if this group is not trivial, it is also free over a generating set which can be effectively characterized and whose cardinality is the cyclomatic number of the graph $\mathbf{G}$.

\section{The MAXimal GROUPS}

Consider two words $w$ and $w^{\prime}$ such that $w \mathcal{D}^{\prime} w^{\prime}$. Theorem 2.1 and its dual characterize whether $w \mathcal{H}^{\prime} w^{\prime}$ in terms of their $\mathcal{R}$-entrances and $\mathcal{L}$-entrances. In fact, they imply that $w \mathcal{R}^{\prime} w^{\prime}$ if and only if $\epsilon_{R}(w) \sim \epsilon_{R}\left(w^{\prime}\right)$ and that $w \mathcal{L}^{\prime} w^{\prime}$ if and only if $\epsilon_{L}(w) \sim \epsilon_{L}\left(w^{\prime}\right)$. In Theorem 5.1 [13] we extend these results to the characterization of words that represent the same element of $\mathcal{M}$. This is made in terms of their fingerprints and of the canonical projection of the free category generated by the fundamental graph $\mathbf{G}$ of the $\mathcal{D}$-class in question onto the free Burnside groupoid satisfying $x^{m}=1$ generated by $\mathbf{G}$.

Theorem 5.1 (Characterization Theorem). Let $w$ and $w^{\prime}$ be two words such that $w \mathcal{H}^{\prime} w^{\prime}$. Then:

$$
w \sim w^{\prime} \text { if and only if } \sigma(w) \cong \sigma\left(w^{\prime}\right) .
$$

In Theorem 5.2 [13] we have a characterization of the $\mathcal{H}$-classes in terms of the fundamental graph; the proof relies on every one of the preceding theorems.

Theorem 5.2 (Theorem on Maximal Groups). Let $n \geq 1$ and $m \geq 1$ and let $A$ be any alphabet. Every maximal group in the free Burnside semigroup satisfying $x^{n}=x^{n+m}$ generated by $A$ is a free Burnside group satisfying $x^{m}=1$. Furthermore, if $m \geq 2$, this Burnside group is free over a generating set whose cardinality is the cyclomatic number of the fundamental graph of the $\mathcal{D}$-class that contains the group.

One can exhibit a generating set for this free Burnside group.

In Proposition 5.3 we have a characterization of the fundamental graph associated with irregular $\mathcal{D}$-classes, which in turn implies that the irregular $\mathcal{H}$-classes of a free Burnside monoid satisfying $x^{n}=x^{n+m}$ generated by the alphabet $A$ are all trivial.

Proposition 5.3. The fundamental graph of an irregular $\mathcal{D}$-class of a free Burnside semigroup satisfying $x^{n}=x^{n+m}$ generated by an alphabet $A$, with $n \geq 1$ and $m \geq 1$, does not have any edge and has a unique vertex. 
$\epsilon_{L} \widetilde{\left(\epsilon_{R}(w)\right)}$

FiguRE 2. Fundamental graph of an irregular element $\widetilde{w}$.

\section{The Relation $\Sigma$}

Given two words $w$ and $u$, we say that $u \in A^{+}$(possibly longer than $w$ ) is a period of $w$ if $w$ is a factor of some power of $u$. In this case, we also say that $i=|u|$ is a period of $w$. A word $w$ is called primitive if $w=u^{k}$ implies $k=1$ and $u=w$. Two words $w$ and $w^{\prime}$ are called conjugate if there exist words $u$ and $v$ such that $w=u v$ and $w^{\prime}=v u$. We define the overlap of two words $u$ and $v$ by: $u \rightleftharpoons v \stackrel{\text { def }}{=} \max (\operatorname{Suf}(u) \cap \operatorname{Pref}(v))$; that is to say, it is the longest suffix of $u$ that is also a prefix of $v$.

For every pair $\tau=(l, s) \in A^{+} \times A^{+}$we say that $l$ is the long of $\tau$ while $s$ is the short of $\tau$, respectively denoted by $l_{\tau}$ and $s_{\tau}$. We define $\Omega$, a relation in $A^{+}$, as follows: $\Omega \stackrel{\text { def }}{=}\{(l, s) \mid s$ is simultaneously a proper prefix and a proper suffix of $l$ and $s^{-1} l$ is an $m$-power $\}$. Each element of $\Omega$ is called a production. For every production $\tau$ we define the basis of $\tau$ by $\operatorname{bas}(\tau) \stackrel{\text { def }}{=}\left(\left|l_{\tau}\right|-\left|s_{\tau}\right|\right) / m$. We also define the left basis of $\tau, \mathrm{b}_{\mathrm{L}}(\tau)$, by the prefix of $l_{\tau}$ of length bas $(\tau)$; and the right basis of $\tau, \mathrm{b}_{\mathrm{R}}(\tau)$, by the suffix of $l_{\tau}$ of length bas $(\tau)$. We can see an example of these definitions in Figure 3.

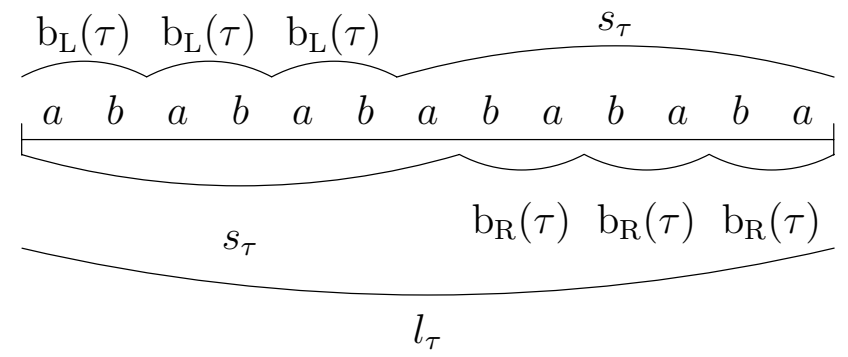

Figure 3. Example of a production with basis 2 for $m=3$.

We define a partial ordering $\preccurlyeq$ in $\Omega$ by $(l, s) \preccurlyeq\left(l^{\prime}, s^{\prime}\right) \stackrel{\text { def }}{\Longleftrightarrow} \exists u, v \in A^{*} \mid l^{\prime}=u l v$ and $s^{\prime}=u s v$. We say that two productions $\tau$ and $\sigma$ are conjugate if $\mathrm{b}_{\mathrm{R}}(\tau)$ and $\mathrm{b}_{\mathrm{R}}(\sigma)$ are conjugate. We say that the production $\tau$ is stable if $\operatorname{bas}(\tau)$ is the smallest period of $s_{\tau}$ and a set of productions is called stable if every production in it is stable. We say that a production $\sigma$ erases a suffix of the word $w$ if $\left|w \rightleftharpoons l_{\sigma}\right|>\left|s_{\sigma}\right|$ and we say that a production $\sigma$ erases a prefix of the word $w$ if $\left|l_{\sigma} \rightleftharpoons w\right|>\left|s_{\sigma}\right|$. 
Let $\Sigma_{0}=\emptyset$. Thus, for every integer $i>0$, we define:

- $\pi_{i}^{\prime}$ is the set of productions of $\pi$ in which: the basis is $i$; the right basis is primitive; their longs do not have factors that are longs of productions in $\Sigma_{i-1}$

- $\Sigma_{i}$ is the set of the productions which are minimal ${ }^{4}$ among all those that are: smaller than or equal to some production of $\pi^{\prime}{ }_{i}$; maximal among those that cannot be erased neither on the right nor on the left by any production of $\Sigma_{i-1}$.

All productions of $\Sigma_{i}$ have basis $i$ and we define

$$
\Sigma=\bigcup_{i=1}^{\infty} \Sigma_{i}
$$

A fundamental property of $\Sigma$ is that it generates the same congruence as $\pi$. Another fundamental property to be investigated for $\Sigma$ is the stability of its productions. We know that $\Sigma$ is not stable neither for $n=1$ nor for $n=2$ and $m \geq 2$ (as we will see in Sect. 9). The stability of $\Sigma$ in the case $n=2$ and $m=1$ remains open. Due to a sequence of results of several authors $[7,8,10-12,17,18,28]$ we know that $\Sigma$ is stable for $n \geq 3$ and $m \geq 1$, as we shall see in Theorem 6.1. The elaboration of $\Sigma$ has started in the works of de Luca and Varricchio $[7,8]$ culminating so far in the results of do Lago $[11,12]$.

Theorem 6.1. Suppose that $n \geq 3$ and $m \geq 1$. Then every production of $\Sigma$ is stable.

In Section 8 we will see what is known about the free Burnside monoid if $\Sigma$ is stable. Even though $\Sigma$ is not stable if $n=1$, we have a complete study of this case due to the fact that we have detailed information on the Green-induced relations and on the free Burnside groups. We will see next the results that are known in the case $n=1$.

\section{THE CASE $n=1$}

We will assume throughout this section that $n=1$ and that $m \geq 1$ is a fixed integer.

A key concept in the study of $\mathcal{M}$ for the cases in which $n=1$ is the content of a word $w$ defined by

$$
c(w) \stackrel{\text { def }}{=} \text { the set of letters of } A \text { that occur in } w .
$$

In particular, due to classical work of Green and Rees [16], all Green-induced relations are characterized and $\mathcal{M}$ has exactly $2^{|A|} \mathcal{D}$-classes because, given words

\footnotetext{
${ }^{4}$ Always using the order $\preccurlyeq$.
} 
$w$ and $w^{\prime}$ it is proved that

$$
w \mathcal{D}^{\prime} w^{\prime} \text { if and only if } c(w)=c\left(w^{\prime}\right) .
$$

Let $k$ be the cardinality of the alphabet $A$. We denote by:

$$
\begin{array}{ll}
D_{k, m} & \text { the cardinality of the } \mathcal{D} \text {-class of } \mathcal{M} \text { the contents of which is } A ; \\
\mathbf{G}_{k, m} & \text { cyclomatic number of the fundamental graph of this } \mathcal{D} \text {-class; } \\
M_{k, m} & \text { the cardinality of } \mathcal{M} \text {; } \\
B(k, m) & \text { the free Burnside group satisfying } x^{m}=1 \text { generated by } A ; \\
B_{k, m} & \text { the cardinality of } B(k, m) .
\end{array}
$$

The characterization of $\mathcal{D}^{\prime}$ and Proposition 3.1 lead us to Theorem 7.1 [13] where we characterize the fundamental graph of a $\mathcal{D}$-class of the free Burnside semigroup satisfying $x=x^{1+m}$. In Figure 4 we see an application of this theorem to the $\mathcal{D}$ class of $\widetilde{a b}$. Note that the cyclomatic number of the fundamental graph of this $\mathcal{D}$-class is $2 m-1$.

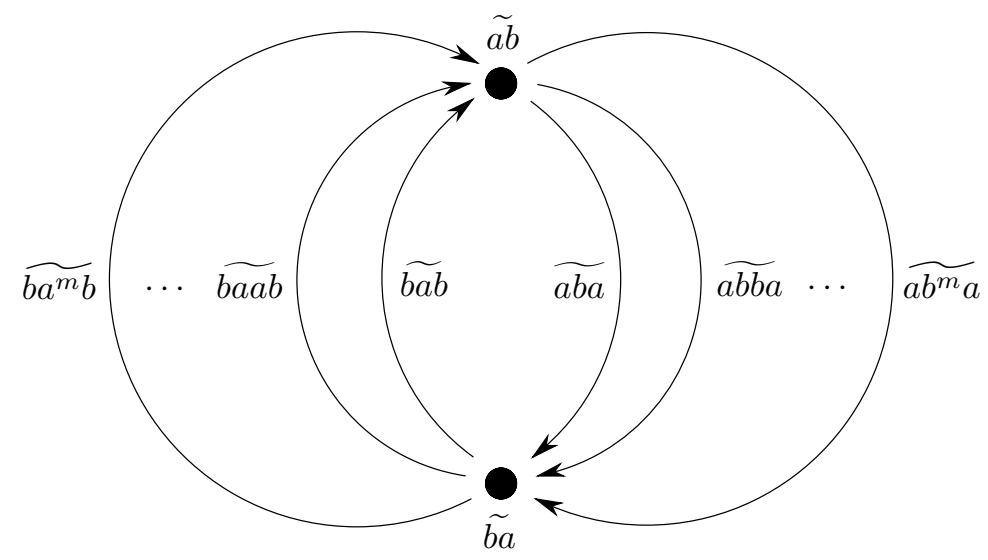

Figure 4. Fundamental graph of the $\mathcal{D}$-class of $\widetilde{a b}$.

Theorem 7.1. Let $m \geq 1$ be an integer, let $A$ be an alphabet, let $D=D(B)$ be the $\mathcal{D}$-class of $\mathcal{M}$ associated with $\emptyset \neq B \subseteq A$ and let $\mathbf{G}$ be the fundamental graph of $D$.

If $B=\{a\}$ then $V(\mathbf{G})=\{\widetilde{a}\}$ and $E(\mathbf{G})=\{\widetilde{a a}\}$. If $|B| \geq 2$, then recalling that $D(\emptyset)=\{\widetilde{1}\}$, we have that

$$
\begin{aligned}
& V(\mathbf{G})=\{\widetilde{a w b} \mid a \in B, b \in B \backslash\{a\}, \widetilde{w} \in D(B \backslash\{a, b\})\}, \\
& E(\mathbf{G})=\{\widetilde{a w a} \mid a \in B, \widetilde{w} \in D(B \backslash\{a\})\} .
\end{aligned}
$$

Furthermore, given $\widetilde{u}, \widetilde{v} \in V(\mathbf{G})$, there exists an edge from $\widetilde{u}$ to $\widetilde{v}$ if and only if the first letter of $u$ is equal to the last letter of $v$. In this case, there exist exactly $B_{\left(\mathbf{G}_{|B|-1, m}\right), m}$ edges from $\widetilde{u}$ to $\widetilde{v}$. 
The characterization of $\mathcal{D}^{\prime}$ and Theorem 2.1 lead us to the fact that the $\mathcal{D}$-class of $\mathcal{M}$ associated to the content $A$ has $k D_{k-1, m} \mathcal{R}$-classes and the same cardinality of $\mathcal{L}$-classes. In Corollary 7.2 [13] we compute the exact cardinality of $\mathcal{M}, M_{|A|, m}$, for every $m$ for which $B_{k, m}$ is known for every $k$. This result extends those of McLean [29].

Corollary 7.2. Let $m \geq 1$ be an integer. Suppose known the cardinality $B_{k, m}$ in function of $k$ and of $m$, for every $k$. Then the following cardinalities can be computed recurrently:

$$
\begin{aligned}
& \mathbf{G}_{k, m}= \begin{cases}1 & \text { if } k=1 ; \\
k D_{k-1, m}-k(k-1) D_{k-2, m}+1 & \text { if } k \geq 2 ;\end{cases} \\
& D_{k, m}= \begin{cases}1 & \text { if } k=0 \\
\left(k D_{k-1, m}\right)^{2} B_{\left(\mathbf{G}_{k, m}\right), m} & \text { if } k \geq 1 ;\end{cases} \\
& M_{k, m}=\sum_{i=0}^{k}\left(\begin{array}{l}
k \\
i
\end{array}\right) D_{i, m} .
\end{aligned}
$$

In Figure 5 we see an application of Theorem 7.1 to the $\mathcal{D}$-class of $\widetilde{a b c}$. Note that the number of vertices of the fundamental graph of this $\mathcal{D}$-class is $6 \mathrm{~m}$ and the number of edges is $12 m^{2} B_{2 m-1, m}$. For $m=1$ and $m=2$ we have cyclomatic numbers 7 and 373, respectively. For the cases in which the free Burnside group with at least 2 generators is infinite ( $m \geq 8000$ for example), $B_{2 m-1, m}$ is infinite

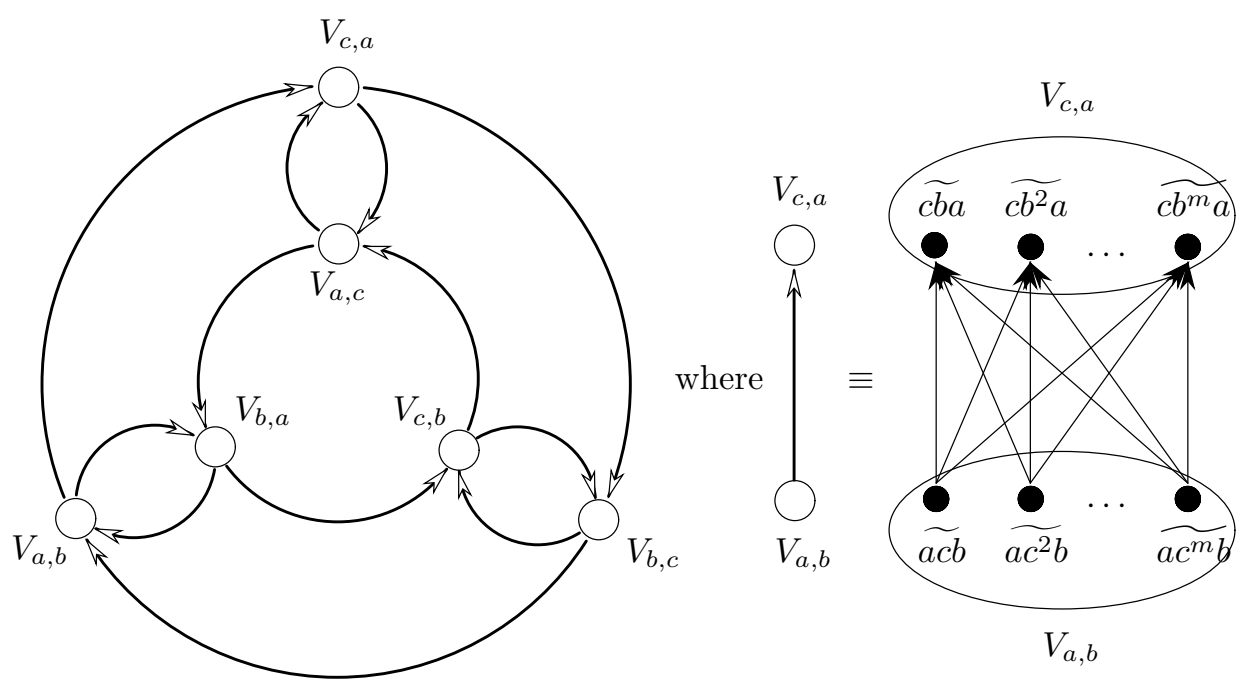

and every edge has multiplicity $B_{2 m-1, m}$

FIGURE 5. Fundamental graph of the $\mathcal{D}$-class of $\widetilde{a b c}$. 
and the cyclomatic number of the fundamental graph in question is infinite. This implies that the $\mathcal{H}$-classes of the $\mathcal{D}$-class of $\widetilde{a b c}$ are infinite free Burnside groups satisfying $x^{m}=1$. These groups would already be infinite if they had only two generators. In this case, even the number of generators of these free Burnside groups is infinite. Since $D_{2, m}$ is infinite, there are infinitely many $\mathcal{R}$-classes and infinitely many $\mathcal{L}$-classes in this $\mathcal{D}$-class, as is shown in Figure 6.

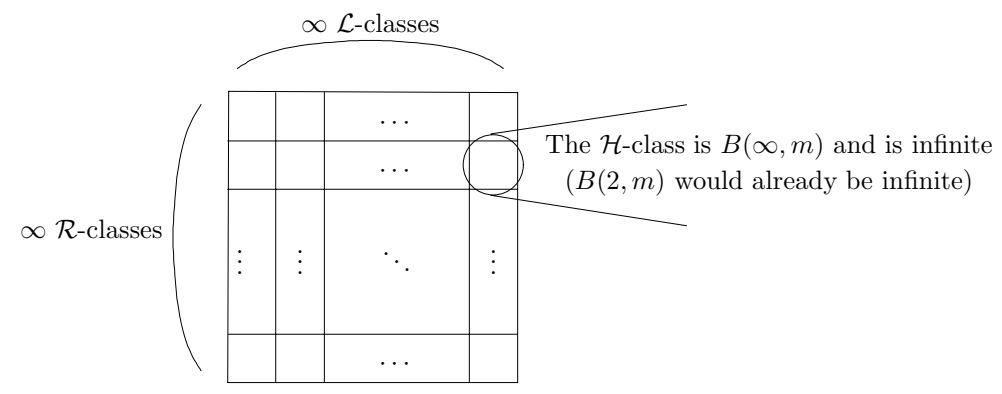

Figure $6 . \mathcal{D}$-class of $\widetilde{a b c}$ for $m$ large enough.

Another consequence of Theorem 7.1 is Corollary 7.3, where we will see a result of Kad̆ourek and Polák [22] that reduces the word problem in $\mathcal{M}$ to the word problem in a free Burnside group $B(k, m)$ for $k$ finite. Recall that the word problem is decidable in $B(k, m)$ whenever $B(k, m)$ is finite. This is the case for $m=1$ (since the groups are trivial); for $m=2$ (due to Burnside [6]); for $m=3$ (due to Burnside [6] and de Levi and van der Waerden [25]); for $m=4$ (due to Sanov [33]); and for $m=6$ (due to Hall [19]). Furthermore, recall that $B(2, m)$ is infinite, but its word problem is decidable in certain circumstances. This is the case for $m \geq 665$ and odd (due to Adyan [1,2]) and for $m \geq 8000$ and multiple of 16 (due to Lysënok [26]). Indeed, Lysënok proves that $B(k, m)$ is infinite for any $m \geq 8000$ and $k \geq 2$, but one does not know whether or not the word problem is decidable in the cases in which $m$ is multiple of 2 but not of 16 . Other important works in the infinite case include those of Novikov and Adyan [30,31], Ol'shanskii [32] and Ivanov [21].

Corollary 7.3. Let $m \geq 1$ be an integer. Suppose the word problem to be decidable in any finitely generated free Burnside group satisfying $x^{m}=1$. Then the word problem is decidable in any finitely generated free Burnside monoid satisfying $x=$ $x^{m+1}$.

\section{The Case Where $\Sigma$ IS Stable}

Contrasting completely with the case $n=1$, where there are exactly $2^{|A|} \mathcal{D}$ classes and the relation $\mathcal{D}^{\prime}$ is completely characterized, using Thue-Morse words [36] and the work of Brzozowski et al. [4], we know that the free Burnside monoids are infinite if $|A| \geq 2$ and $n \geq 2$, and that they have infinitely many $\mathcal{D}$-classes. 
Nevertheless, the fact that $\Sigma$ is stable simplifies substantially the investigation of these monoids, resulting in a relatively simple structure which can be described precisely. For example, as will be seen in Theorem 8.2, the fundamental graph of any regular $\mathcal{D}$-class of $\mathcal{M} \backslash\{\tilde{1}\}$ is a cycle.

All the results of this section rely exclusively on the hypothesis that $\Sigma$ is stable (recall Th. 6.1), and they do not depend further on the values of $n, m$ and $|A|$.

We say that a word $w$ contracts to a word $w^{\prime}$ using a production $\tau$ if $w^{\prime}$ can be obtained from $w$ by substituting an occurrence of $l_{\tau}$ by $s_{\tau}$. We will use the terminology of rewriting systems for a while and one can find the definitions in [20, 23]. Suppose that $\Sigma$ is stable. An important property that can be proved is that the stability of $\Sigma$ implies that $\Sigma$ is locally confluent. Figure 7 shows this situation where each arrow represents a contraction (reduction) using the labeled production. This leads to Theorem 8.1 and implies that $\Sigma$ is a Church-Rosser rewriting system. Moreover, we can effectively solve the word problem for the congruence $\sim$, and one can also prove that $\Sigma$ is a minimal ${ }^{5}$ generator of $\sim$.

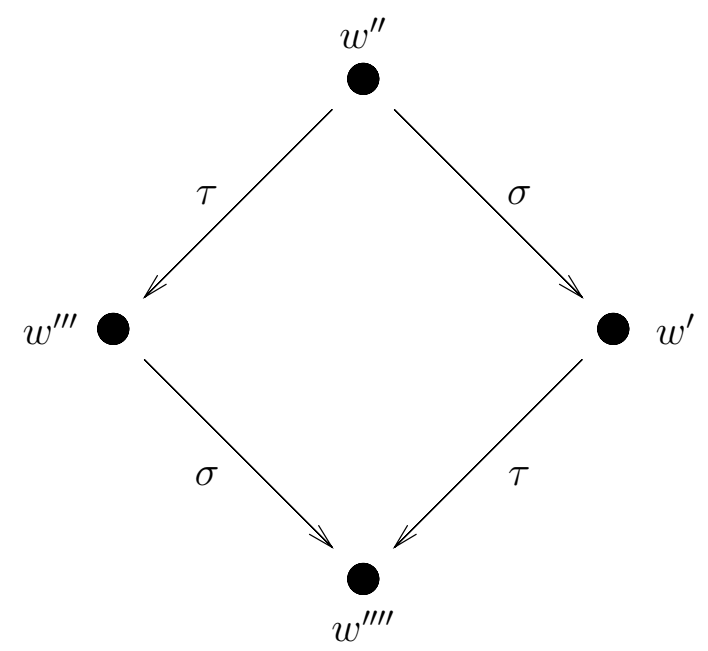

FiguRE $7 . \quad \Sigma$ is locally confluent.

Theorem 8.1 (Expansiveness Theorem). Assume that $\Sigma$ is stable. Then each congruence class of $\sim$ has a unique word which does not have any factor which is a long of a production of $\Sigma$. Further, such a word is the unique shortest word in its class.

Applying Theorem 8.1, we can define the representative of a given word $w$, by $\operatorname{rep}(w) \stackrel{\text { def }}{=}$ the shortest word of $\widetilde{w}$, and this leads us to a useful characterization

${ }^{5}$ With respect to the order of subsets. 
of the multiplication in $\mathcal{M}$ : for $a \in A$ and $u=\operatorname{rep}(w)$ we have that $\operatorname{rep}(u a) \neq u a$ if and only if there exists $\sigma \in \Sigma$ such that $l_{\sigma} \in \operatorname{Suf}(u a)$, in which case $\operatorname{rep}(u a)=$ $\left(u a l_{\sigma}^{-1}\right) s_{\sigma}$.

We can also compute the fundamental graph of the regular $\mathcal{D}$-classes as is done in Theorem 8.2. Hence, applying Theorem 5.2 we obtain Corollary 8.3.

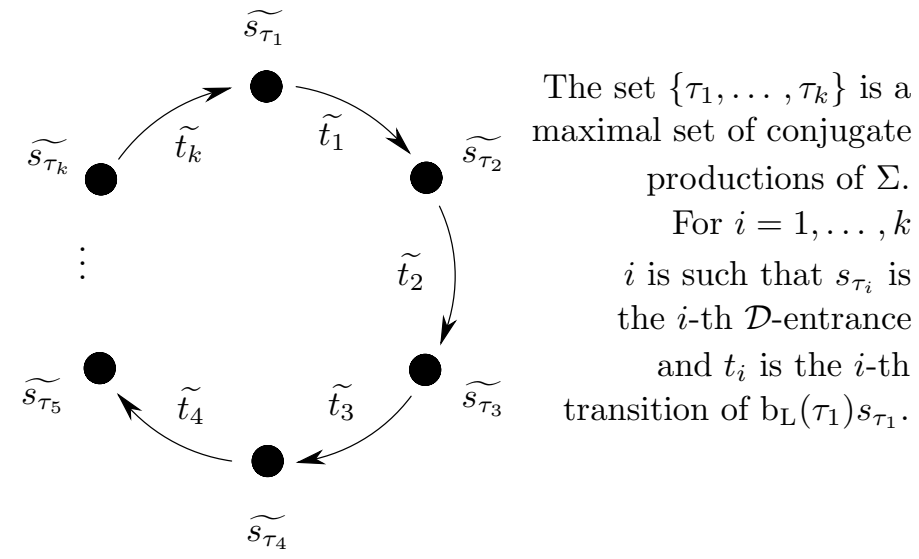

Figure 8. Fundamental Graph of a regular $\mathcal{D}$-class for $\Sigma$ stable.

Theorem 8.2. Assume that $\Sigma$ is stable. The fundamental graph of any regular $\mathcal{D}$-class of the free Burnside semigroup satisfying $x^{n}=x^{n+m}$ is a cycle as in Figure 8 .

Corollary 8.3. Assume that $\Sigma$ is stable. The maximal groups in the free Burnside semigroup satisfying $x^{n}=x^{n+m}$ are cyclic of order $m$.

Given words $w$ and $w^{\prime}$ such that $w \mathcal{R}^{\prime} w^{\prime}$, a consequence of Theorem 2.1 is that $\epsilon_{R}(\operatorname{rep}(w))=\epsilon_{R}\left(\operatorname{rep}\left(w^{\prime}\right)\right)=\operatorname{rep}\left(\epsilon_{R}(w)\right)$. Hence we can define the $\mathcal{R}$-cactus of $w$ by the set

$$
\operatorname{cactus}_{\mathcal{R}}(w) \stackrel{\text { def }}{=}\left\{\operatorname{rep}\left(\epsilon_{R}(w)\right)^{-1} \operatorname{rep}\left(w^{\prime}\right) \mid w^{\prime} \mathcal{R}^{\prime} w\right\}
$$

Note that any $\mathcal{R}$-cactus is closed by prefixes. Dually, define $\operatorname{cactus}_{\mathcal{L}}(w) \stackrel{\text { def }}{=}$ $\left\{\operatorname{rep}\left(w^{\prime}\right) \operatorname{rep}\left(\epsilon_{L}(w)\right)^{-1} \mid w^{\prime} \mathcal{L}^{\prime} w\right\}$. We can prove that the $\mathcal{R}$-cactus of $w$ is the $\mathcal{R}$ cactus of the longest short of a production of $\Sigma$ which is a $\operatorname{suffix}$ of $\operatorname{rep}\left(\epsilon_{R}(w)\right)$. Furthermore, we can compute recursively on $\operatorname{bas}(\tau)$ all the $\mathcal{R}$-cacti of shorts of productions of $\Sigma$, and they are all finite. Hence, all $\mathcal{R}$-classes are finite, and dually, all $\mathcal{L}$-classes are finite.

This leads us to the following theorem, which settles a conjecture of Brzozowski [3]:

Theorem 8.4. If $\Sigma$ is stable, then $\mathcal{M}$ is finite $\mathcal{J}$-above and every congruence class is recognizable. 
All results presented in this section do not depend on particular values of $n, m$ or $|A|$ but only on the stability of $\Sigma$. They are from [12], where one can find the details and more structural results. The unique exception is Theorem 8.2 which comes from [13].

\section{THE CASE $n=2$}

The main result in this section is Corollary $9.2[13,14]$ which states that we have noncyclic maximal groups in $\mathcal{M}$ for $n=2$ and $m \geq 2$. In some sense, the case $n=2$ simultaneously presents the complexities of the cases $n=1$ and $n \geq 3$. While the maximal groups of $\mathcal{M} \backslash\{\widetilde{1}\}$ are cyclic of order $m$ for $n \geq 3$, they can have more generators and can be infinite for $n \leq 2$. While there are exactly $2^{|A|}-1 \mathcal{D}$-classes which in turn are easily characterized in the case $n=1$, there are infinitely many $\mathcal{D}$-classes which are in turn difficult to characterize for $n \geq 2$.

Indeed, the case $n=2$ was the last one to be analysed, and our knowledge in this case is still quite incomplete. For instance, whether or not $\Sigma$ is stable for $n=2$ and $m=1$ is an open problem. If it holds, all the nice properties of Section 8 follow. On the other hand, $\Sigma$ is not stable for $n=2$ and $m \geq 2$. Indeed, let $x=\left(b(a b)^{m+1}\right)$ and let $\sigma=\left((a b)^{2} x^{m}(b a)^{2},(a b)^{2}(b a)^{2}\right)$. One can verify that $\sigma \preccurlyeq\left(x^{2+m}, x^{2}\right)$ is an unstable production of $\Sigma$.

In Theorem $9.1[13,14]$ we investigate the fundamental graph of the $\mathcal{D}$-class of $(\widetilde{b a b a b})^{2}$ :

Theorem 9.1. Assume that $n=2$ and $m \geq 2$. Assume that $A=\{a, b\}$. Then the graph $\mathbf{G}$ presented on Figure 9 is a subgraph of the fundamental graph of the $\mathcal{D}$-class of $(\widetilde{b a b a b})^{2}$.

The next result $[13,14]$ reveals important structural information regarding $\mathcal{M}$ and in particular it shows that Brzozowski's conjecture [3] does not hold for $n=2$ and $m \geq 8000$.

Corollary 9.2. The maximal groups in the $\mathcal{D}$-class of $\left(\widetilde{\text { babab })^{2}}\right.$ are free Burnside groups satisfying $x^{m}=1$ with at least $2 m-1$ generators. For $m$ sufficiently large, these groups are infinite and the congruence classes of $A^{*}$ projected onto the elements of this $\mathcal{D}$-class are not recognizable.

Let $n=2$ and $m=2$ and let $\mathbf{G}$ be the graph defined in Figure 9. Let $p=$ $(\widetilde{A}, \widetilde{B}, \widetilde{C})$ and $q=\left(\widetilde{D_{1}}\right)$ be two paths in $\mathbf{G}$. One can prove that the shortest words that have fingerprints $p q$ and $q p$, respectively, are congruent and both are shortest words in the same congruence class. This fact contrasts with the situation seen in Theorem 8.1. 


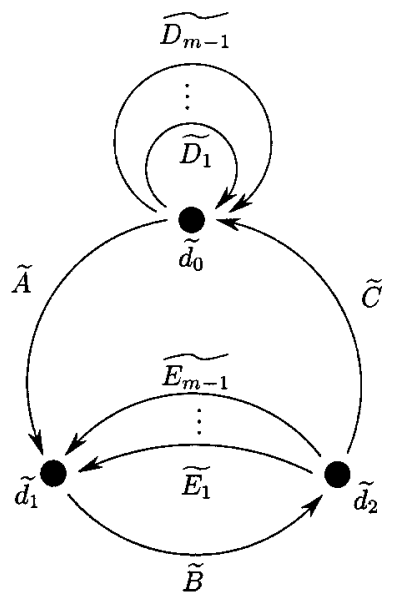

The transitions: The $\mathcal{D}$-entrances:

$$
\begin{array}{lll}
A: & a 35 b a & d_{0}: a 33 a \\
B: & 353 & d_{1}: 35 b a \\
C: & a b 53 a & d_{2}: a b 53 \\
D_{1}: & a 373 a & \\
\vdots & \\
D_{m-1}: & a 35(a b)^{m-1} 3 a \\
E_{1}: & a b 535 b a \\
\vdots & \\
E_{m-1}: & a b 53^{m-1} 5 b a
\end{array}
$$

Where: 3 denotes $b a b$

5 denotes $b a b a b$

7 denotes $b a b a b a b$

Figure 9. The graph G.

\section{REFERENCES}

[1] S.I. Adian, The Burnside problem and identities in groups. Springer-Verlag, Berlin-New York, Ergebnisse der Mathematik und ihrer Grenzgebiete 95 [Results in Mathematics and Related Areas] (1979). Translated from the Russian by John Lennox and James Wiegold.

[2] S.I. Adyan, The Burnside problem and identities in groups. Izdat. "Nauka", Moscow (1975).

[3] J. Brzozowski, Open problems about regular languages, edited by R.V. Book. Academic Press, New York, Formal Language Theory, Perspectives and Open Problems (1980) 23-47.

[4] J. Brzozowski, K. Čulk and A. Gabrielian, Classification of non-counting events. J. Comput. System Sci. 5 (1971) 41-53.

[5] J.A. Brzozowski and I. Simon, Characterizations of locally testable events. Discrete Math. 4 (1973) 243-271.

[6] W. Burnside, On an unsettled question in the theory of discontinuous groups. Quart. J. Math. 33 (1902) 230-238.

[7] A. de Luca and S. Varricchio, On non-counting regular classes, edited by M.S. Paterson, Automata, Languages and Programming. Springer-Verlag, Berlin, Lecture Notes in Comput. Sci. 443 (1990) 74-87.

[8] A. de Luca and S. Varricchio, On non-counting regular classes. Theoret. Comput. Sci. 100 (1992) 67-104.

[9] A.P. do Lago, Local groups in free groupoids satisfying certain monoid identities (to appear).

[10] A.P. do Lago, Sobre os semigrupos de Burnside $x^{n}=x^{n+m}$, Master's Thesis. Instituto de Matemática e Estatística da Universidade de São Paulo (1991).

[11] A.P. do Lago, On the Burnside semigroups $x^{n}=x^{n+m}$, in LATIN'92, edited by I. Simon. Springer-Verlag, Berlin, Lecture Notes in Comput. Sci. 583 (1992) 329-343.

[12] A.P. do Lago, On the Burnside semigroups $x^{n}=x^{n+m}$. Int. J. Algebra Comput. 6 (1996) 179-227.

[13] A.P. do Lago, Grupos Maximais em Semigrupos de Burnside Livres, Ph.D. Thesis. Universidade de São Paulo (1998). Electronic version at <http://www.ime.usp.br/ ${ }^{\sim}$ alair/Burnside>

[14] A.P. do Lago, Maximal groups in free Burnside semigroups, in LATIN'98, edited by C.L. Lucchesi and A.V. Moura. Springer-Verlag, Berlin, Lecture Notes in Comput. Sci. 1380 (1998) 70-81. 
[15] S. Eilenberg, Automata, languages, and machines, Vol. B. Academic Press [Harcourt Brace Jovanovich Publishers], New York (1976). With two chapters ("Depth decomposition theorem" and "Complexity of semigroups and morphisms") by B. Tilson, Pures Appl. Math. 59.

[16] J.A. Green and D. Rees, On semigroups in which $x^{r}=x$. Proc. Cambridge. Philos. Soc. 48 (1952) 35-40.

[17] V.S. Guba, The word problem for the relatively free semigroup satisfying $t^{m}=t^{m+n}$ with $m \geq 3$. Int. J. Algebra Comput. 2 (1993) 335-348.

[18] V.S. Guba, The word problem for the relatively free semigroup satisfying $t^{m}=t^{m+n}$ with $m>4$ or $m=3, n=1$. Int. J. Algebra Comput. 2 (1993) 125-140.

[19] M. Hall, Solution of the Burnside problem for exponent six. Illinois J. Math. 2 (1958) 764-786.

[20] G. Huet and D.C. Oppen, Equations and rewrite rules: A survey, edited by R.V. Book. Academic Press, New York, Formal Language Theory, Perspectives and Open Problems (1980) 349-405.

[21] S.V. Ivanov, The free Burnside groups of sufficiently large exponents. Int. J. Algebra Comput. 4 (1994) ii +308.

[22] J. Kadourek and L. Polák, On free semigroups satisfying $x^{r} \simeq x$. Simon Stevin 64 (1990) 3-19.

[23] J.W. Klop, Term rewriting systems: From Church-Rosser to Knuth-Bendix and beyond, edited by M.S. Paterson, Automata, Languages and Programming. Springer-Verlag, Berlin, Lecture Notes in Comput. Sci. 443 (1990) 350-369.

[24] G. Lallement, Semigroups and Combinatorial Applications. John Wiley \& Sons, New York (1979).

[25] F.W. Levi and B.L. van der Waerden, Über eine besondere Klasse von Gruppen. Abh. Math. Sem. Hamburg 9 (1933) 154-158.

[26] I.G. Lysënok, Infinity of Burnside groups of period $2^{k}$ for $k \geq 13$. Uspekhi Mat. Nauk 47 (1992) 201-202.

[27] S. MacLane, Categories for the working mathematician. Springer-Verlag, New York, Grad. Texts in Math. 5 (1971).

[28] J. McCammond, The solution to the word problem for the relatively free semigroups satisfying $t^{a}=t^{a+b}$ with $a \geq 6$. Int. J. Algebra Comput. 1 (1991) 1-32.

[29] D. McLean, Idempotent semigroups. Amer. Math. Monthly 61 (1954) 110-113.

[30] P.S. Novikov and S.I. Adjan, Infinite periodic groups. I. Izv. Akad. Nauk SSSR Ser. Mat. 32 212-244.

[31] P.S. Novikov and S.I. Adjan, Infinite periodic groups. II. Izv. Akad. Nauk SSSR Ser. Mat. 32 (1968) 251-524.

[32] A.Y. Ol'shanskiı̌, Geometry of defining relations in groups. Kluwer Academic Publishers Group, Dordrecht (1991). Translated from the 1989 Russian original by Yu.A. Bakhturin.

[33] I. Sanov, Solution of Burnside's problem for exponent 4. Leningrad. Gos. Univ. Uchen. Zap. Ser. Mat. 10 (1940) 166-170 (Russian).

[34] I. Simon, Notes on non-counting languages of order 2. Manuscript (1970).

[35] H. Straubing, Finite automata, formal logic, and circuit complexity. Birkhäuser Boston Inc., Boston, MA (1994).

[36] A. Thue, Über die gegenseitige Lage gleicher Teile gewisser Zeichenreihen. Norske Vid. Selsk. Skr. I Mat. Nat. Kl. 1 (1912) 1-67.

[37] B. Tilson, Categories as algebra: An essential ingredient in the theory of monoids. J. Pure Appl. Algebra 48 (1987) 83-198.

Received March 27, 2001. Revised January 31, 2002.

To access this journal online: www.edpsciences.org 